\title{
Recovery from latent extinction
}

WAYNE J. WILSON AND THOMAS A. PETERS STEPHEN F. AUSTIN STATE COLLEGE

A 2 by 5 design was utilized to investigate two levels of reward magnitude under conditions including the administration of nonrewarded placements to rats in the start box, mid box, and goal box of a straight runway, with two conditions used as controls for the latent extinction effect. High reward Ss showed faster recovery of start times under partial reinforcement but did not differ from low reward Ss in a prior stage of $100 \%$ reward. Nonplacement control Ss displayed faster recovery under partial reward than did $S s$ in the latent extinction conditions.

Latent extinction (LE) has been identified operationally as the act of placing an organism in commerce with formerly rewarded stimuli in order to produce a performance decrement on subsequent instrumental trials. Wilson \& Dyal (1963) and Dyal (1965) have demonstrated that partial reinforcement $(P R)$ tends to reduce the decremental effects of pre-extinction placements on running times during the first regular extinction trial. Although LE effects have been indexed most clearly by slower goal box times in the initial post-placement test trial (Dyal, 1964), this decremental influence is obscured rapidly when Ss are exposed to an ongoing number of regular extinction trials.

By presenting a daily pattern of consecutive nonrewarded placements contiguous with a rewarded running trial, it would seem feasible that this PR procedure should permit recovery from LE to be prolonged and capable of analysis over a number of days. Two variables, reward magnitude (RM) and locus of nonreward $(\mathrm{LN})$, were selected as agents which might influence recovery from LE under the PR condition described. Method

The Ss were 130 female Sprague-Dawley rats obtained from a commercial supplier at 90 days of age. Animals were 100-130 days old and weighed within a range of 225-275 $\mathrm{g}$ at the time of experimentation. Eighteen Ss were eliminated due to illness and $E$ error and were replaced by random selection from the remaining pool of animals.

An unpainted straight alley, $64 \times 51 / 4 \times 41 / 8$ in., contained three $12 \times 51 / 4 \times 4$ in. inserts of 24 ga. sheet metal painted flat white, gray, or black and designed to fit in the start, middle, and goal box locations. Goal box inserts housed a 1-1/2 in. shield behind which a recessed food cup was attached. Starting times (ST) were measured from release of the start gate until $S$ crossed a photobeam 2 in. beyond the entrance. (A detailed account of the methodology and the remaining runway measures can be obtained from the senior author on request.)

The 130 Ss were assigned randomly to 10 treatments which composed a 2 by 5 design. Values for RM were either $10 \quad 45 \mathrm{~g}$ Noyes pellets or one pellet given on each rewarded trial. Conditions for $\mathrm{LN}$ consisted of four nonrewarded placements given, respectively, in the start box (SB), mid box (MB), goal box (GB), or neutral box (NB). Rats in a fifth condition (PR) received four nonrewarded runs (rather than placements) to the goal box and were confined there for $60 \mathrm{sec}$, a detainment period comparable to that experienced by goalbox placed Ss (Group GB-10 and Group GB-1).

Pretraining Day 1 consisted of 5 min solo exploration; on Day 2 each $\mathrm{S}$ was placed in the goalbox until 1 min of eating time was accumulated from a Purina block pellet, or until 5 min confinement time had elapsed. Stage 1 (Training Days 1-8) introduced $R M$ in which all Ss received five massed trials daily, under $100 \%$ reward, with $1-2 \mathrm{~min}$ intertrial intervals. Stage 2 (Days 9-17) introduced the LN variable where four consecutive nonrewarded exposures were given each $S$, followed by a single rewarded run to the goal box in accordance with the appropriate RM value. Placement duration, interplacement intervals, and placement-trial intervals were designated at $60 \mathrm{sec}$. Stage 3 consisted of regular extinction trials except that on Test Day 1 a block of 10 massed extinction runs was preceded by a final pattern of four nonrewarded exposures.

\section{Resulis and Discussion}

Starting latencies were the most sensitive measure taken of the five runway times, therfore, only ST data are reported here. Despite the 10- vs 1-pellet RM values which existed during Stage 1, no significant differences were found between 10- and 1-pellet Ss after an accumulation of 40 rewarded trials on Day 8 $(F<1)$. The initial $L E$ effect on Day 9 was evaluated by using difference scores derived from Day 8 minus Day 9 reciprocal measures, with these data offering the only nonconfounded assessment of placement effects during Stage 2. A 2 by 5 analysis of these scores revealed the continued absence of $\mathrm{RM}$ effects on Day 9 , although statistically reliable effects were found for LN conditions $(F=10.74$, df $=4 / 120, p<.01)$. Tukey's conservative test (Winer, 1962, p. 88 ) indicated that all significant $L N$ results could be accounted for by a comparison of certain LE conditions (Groups SB-10, SB-1, MB-10, GB-1) with NB controls. The initial LE effect produced no significant differences between LE conditions, nor was there a strong interaction effect between RM and LN variables.

Figure 1 confirms these results by showing that RM does not become manifest until Day 11, wherein 10pellet Ss display faster recovery of starting times than 1-pellet Ss. Figure 2 exhibits no appreciable differences 


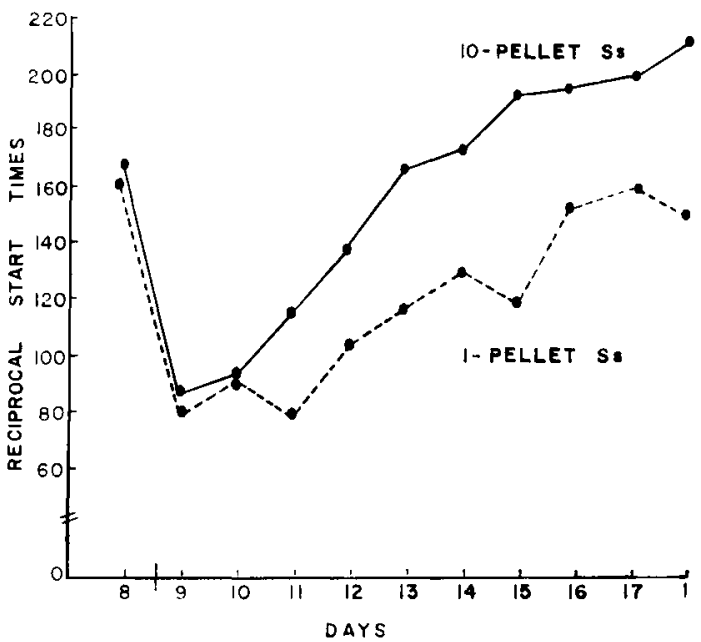

Fig. 1. Faster recovery of starting times shown by 10-pellet Ss. Test day 1 is included since the initial block of five trials was comparable to that given during Days 9-17.

between LE groups throughout Stage 2 but an accelerated recovery is depicted for PR Ss. Note also that NB Ss are displaying faster starting times under the $P R$ schedule that under $100 \%$ reward (Day 8 ).

A 2 by 5 trend analysis of recovery data (Fig. 1) yielded a significant interaction effect between RM

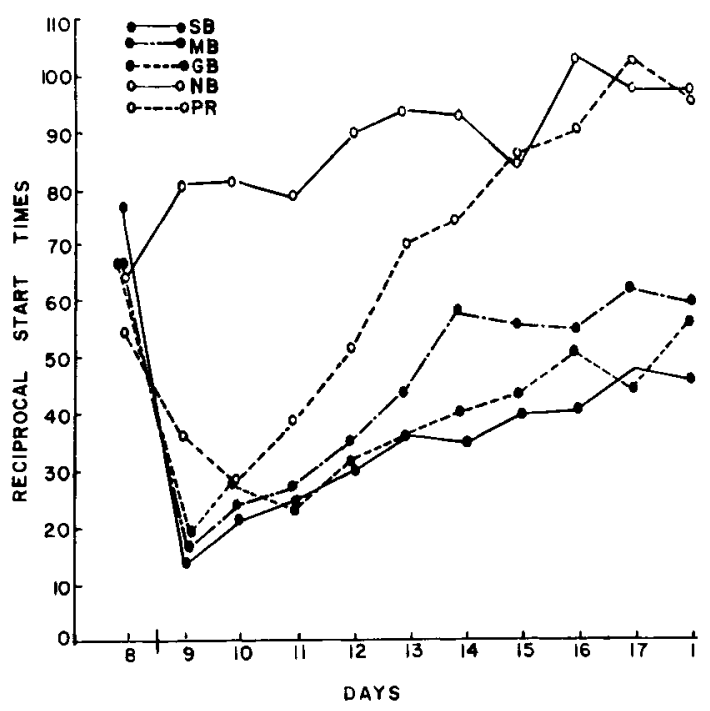

Fig. 2. Faster recovery of starting times for PR Ss in comparison to Placement Groups SB, MB, and GB. Note faster starting times for NB control Ss under partial reinforcement than under $100 \%$ reward on Day 8. and the days comprising Stage 2 ( $F=4.63, \mathrm{df}=9 / 1080$, $p<.01$ ); however, the interaction between $L N$ and the days' variable represented in Fig. 2 was not reliable statistically.

The present design offered qualitatively different exposures to a frustrative event (Rf). Consider the startbox confined $S$ which is frustrated by its inability to gain release from this enclosure, then speculate on the goal box detained $S$ which should become aroused emotionally due to the absence of reward. Day 9 data would lead one to construe that the type of Rf aroused in the start box is just as debilitating on starting times as the mode of Rf displayed in the goal box. Presumably as repetition of $\mathrm{Rf}$ continues under a $\mathrm{PR}$ schedule, the drive function of $\mathrm{Rf}$ (frustration effect or $\mathrm{FE}$ ) will overcome inhibitory influences when $\mathrm{rf}$-sf become associated with rg-sg in conditioning $S$ to enter the end box, despite the presence of aversive $\mathrm{Rf}$ stimuli.

If it is assumed that limited movements condition $S$ to generalize the Rf event less effectively (Trapold \& Doren, 1966; Trapold \& Holden, 1966), then FE would be reduced, and the slower recovery rate of LE Ss in Fig. 2 could be explained. The global locomotor chain exercised more frequently by PR Ss allowed them to demonstrate greater FE by means of faster starting times. However, the conflict between $\mathrm{rg}$ and $\mathrm{rf}$ under limited locomotion would have to generalize quickly to impair starting times and account for the initial LE effect derived from various loci of nonreward on Day 9 . If so, it must be assumed that the inhibitory effects of $\mathbf{r f - s f}$ are capable of rapid development when only a fractional nonrewarded replication of the locomotor chain is permitted, but that the motivational component of $\mathrm{Rf}$ is attenuated when movements are confined.

References

DYAL, J. Latent extinction as a function of placement-test interval and irrelevant drive. J. exp. Psychol., 1964, 68, 486-491.

DYAL, J. Latent extinction as a function of partial reinforcement of the running response. Psychon. Sci., 1965, 3, 207-208.

TRAPOLD, M., \& DOREN, D. Effect of noncontingent partial reinforcement on the resistance to extinction of a runway response. $J$. exp. Psychol., 1966, 71, 429-431.

TRAPOLD, M., \& HOLDEN, D. Noncontingent partial reinforcement of running: A replication. Psychon. Sci., 1966, 5, 449-450.

WILSON, W., \& DYAL, J. Effects of nonresponse acquisition on latent extinction in a differential reinforcement setting. Psychol. Rep., $1963,13,879-884$.

WINER, B. Statistical principles in experimental design. New York: MoGraw-Hill, 1962.

Note

1. This research was supported by the National Institute of Mental Health, MH-12274-01. 